\title{
Hemodiálisis en Pacientes con Mieloma Múltiple: a propósito de dos casos
}

\author{
Elena Garza Jiménez, Dolores Piña Simón, Ide Margarita Rojas Alfaro
}

Unidad de Diálisis. FRIAT- Hospital Rey Juan Carlos. Móstoles. Madrid. España

\section{Resumen}

El Mieloma múltiple es una patología hematológica en la que existe una proliferación de células plasmáticas a nivel de la médula ósea, causan un exceso de producción de cadenas ligeras, la presencia de estas cadenas ligeras libres incompletas es la causa principal de insuficiencia renal.

Caso clínico: Presentamos dos casos de pacientes de 56 y 73 años con mieloma múltiple con cadenas Lambda y Kappa y con fracaso renal agudo. Fueron tratados en la unidad de diálisis del Hospital Rey Juan Carlos con hemodiálisis largas utilizando el filtro Filtryzer BK$2.1 \mathrm{~F}^{\circledR}$.

Ambos precisaron de terapia combinada de quimioterapia y hemodiálisis largas y diarias con dializador de poro ancho. Se apreció que con las sesiones de hemodiálisis las cadenas ligeras disminuyeron considerablemente $y$ que los dos pacientes mejoraron el filtrado glomerular sin precisar sesiones de hemodiálisis. La complicación más frecuente fue la coagulación del circuito extracorpóreo.

Se resalta la importancia iniciar un diagnóstico y tratamiento precoz para reducir el riesgo de insuficiencia renal crónica.

PALABRAS CLAVES: dializador hight cut-off; cadenas ligeras; mieloma múltiple; hemodiálisis; insuficiencia renal aguda; caso clínico.

\section{Correspondencia:}

Elena Garza Jiménez

Unidad de Diálisis. FRIAT. Hospital Rey Juan Carlos

C/ Gladiolo s/n. 28933 Móstoles. Madrid

E-mail: ele_g_j@hotmail.com

elena.garza@hospitalreyjuancarlos.es

\section{Hemodialysis in Patients with Multiple Myelo- ma: A report of two cases}

\begin{abstract}
Multiple myeloma is a hematological pathology characterized by a proliferation of plasma cells in the bone marrow, causing an excess production of free light chains, being this fact the main cause of renal failure.

Case report: We present two cases of patients aged 56 and 73 years with multiple myeloma with Lambda and Kappa chains and with acute renal failure. They were treated in the dialysis unit of the Rey Juan Carlos Hospital with long-term hemodialysis using the Filtryzer BK-2.1F filter.
\end{abstract}

Both required combined daily and long-term chemotherapy and hemodialysis with a wide-pore dialyzer. It was observed that with the hemodialysis sessions the light chains diminished considerably and that the two patients improved the glomerular filtration rate without needing hemodialysis sessions. The most frequent complication was the coagulation of the extracorporeal circuit.

The importance of initiating early diagnosis and treatment to reduce the risk of chronic renal failure is highlighted.

KEYWORDS: high cut-off dialyzer; free light chains; multiple myeloma; hemodialysis; acute renal failure; case report. 


\section{Introducción}

El mieloma múltiple es una patología hematológica cuya frecuencia se estima en 4,3 casos por cada cien mil personas por año y se presenta sobre todo en pacientes mayores de 65 años con predominio del sexo masculino $0^{1,2}$.

Consiste en una proliferación atípica de células plasmáticas en la médula ósea que producen y secretan una inmunoglobulina anómala a la sangre. Cada inmunoglobulina tiene una estructura específica con dos componentes principales: cadenas pesadas y cadenas ligeras, que se ensamblan en una única molécula. Hay cinco tipos posibles de cadenas pesadas, que se definen con letras $G, A, D, E$ y $M$, y dos tipos de cadenas ligeras que se marcan con las letras griegas Kappa y Lambda. Cada inmunoglobulina individual ( Ig) puede tener únicamente uno de los tipos de cadenas pesadas y uno de los tipos de cadenas ligeras.

En el mieloma, solo existe una única combinación de cadena pesada y cadena ligera, la proteína monoclonal. Los mielomas más frecuentes son del tipo IgG. Esto significa inmunoglobulina de tipo $\mathrm{G}$ (uno de los tipos de cadena pesada posibles), en combinación uno de los tipos de cadena ligera, Kappa o Lambda. El segundo tipo más común es el mieloma IgA, también en combinación con cualquiera de las dos cadenas ligeras.

Aproximadamente el $30 \%$ de los pacientes al mismo tiempo que producen una estructura completa de inmunoglobulina producen también cadenas ligeras en exceso, que no se acoplan a una cadena pesada y que se eliminan por la orina donde se detectan con más precisión que en la sangre. En el $20 \%$ de los pacientes, las células afectadas por el mieloma producen únicamente cadenas ligeras y nunca llegan a producir cadenas pesadas. Este último caso es el que se conoce como mieloma de cadenas ligeras o de Bence Jones. Este tipo de mielomas son los más propensos a causar daños al riñón y a depositar componentes de la cadena ligera en los riñones, sistema nervioso u otros órganos ${ }^{3,4}$.

La insuficiencia renal es una complicación frecuente y severa en pacientes con mieloma múltiple y suele asociarse a un mal pronóstico ${ }^{5-8}$. El daño se debe, en general, a la acumulación y precipitación de cadenas ligeras en los túbulos distales formando cilindros y obstruyendo la luz del túbulo. Asimismo, las cadenas ligeras presentan toxicidad directa sobre los túbulos proximales, contribuyendo al daño renal. La nefropatía por cilindros es la causa más frecuente de fracaso renal, ocurre en más de la mitad de los afectados y en la mayor parte de los casos requiere de técnicas dialíticas5. Se plantea la necesidad de aplicar una terapia conjunta y simultánea. Por un lado, reducir las cadenas ligeras en la sangre a través de eliminación directa con HD y por otro la de aplicar quimioterapia para eliminar la producción de células secretoras de dichas sustancias.

Los métodos clásicos de depuración extracorpórea como la hemodiálisis convencional o la plasmaféresis no permiten un aclaramiento eficaz de las cadenas ligeras. La hemodiafiltración con reinfusión endógena utiliza un dializador de doble cámara y parece útil en el mieloma con cadena tipo Kappa pero no en la Lambda². La diálisis con filtro de alto cut-off: $\mathrm{HCO}$, que está constituido por una membrana especial cuyo tamaño del poro es el triple del de dializador convencional se ha mostrado eficaz para eliminar las cadenas Kappa y Lambda ${ }^{5-7}$.

Estas membranas con alta permeabilidad son eficaces para eliminar hasta el $90 \%$ de cadenas ligeras durante sesiones prolongadas de 6 a 8 horas diarias. Con este tipo de filtro además de eliminar las cadenas se elimina albúmina, que se debe suplir mediante su administración. Se aconseja la realización de hemodiálisis diaria durante 6 días para luego continuar con días alternos hasta alcanzar niveles de cadenas ligeras inferiores a $500 \mathrm{mg} / \mathrm{l}$ o la recuperación de la función renal. Los principales inconvenientes de estas las membranas es que producen una pérdida sustancial de albúmina y su elevado precio ${ }^{8-12}$.

Presentamos dos casos clínicos de pacientes con mieloma múltiple y fracaso renal agudo con cadenas Kappa y Lambda. Fueron tratados en la unidad de diálisis del Hospital Rey Juan Carlos (Móstoles) con hemodiálisis, utilizando filtro Filtryzer BK-2.1F ${ }^{\circledR}$ Hight cut-off de doble poro de fibra de polimetilmetacrilato; es una membrana biocompatible que permite una menor activación del complemento y está esterilizada por rayos gamma.

\section{Caso 1}

Varón de 56 años, sin antecedentes personales de interés. Acude al servicio de urgencias por astenia, sensación de plenitud y falta de apetito desde hace 3 días, no presenta fiebre ni disminución de peso.

El 1 agosto 2016 ingresa para estudio de sospecha, según parámetros analíticos, de insuficiencia renal. Cinco 
días más tarde, se inicia tratamiento de hemodiálisis, y se realizan pruebas complementarias. En la gastroscopia se observa a nivel de estómago que en la mucosa hay una mínima lesión eritematosa sin datos de sangrado y en el antro pilórico un leve eritema. En la analítica de sangre se observa: hemoglobina $8,6 \mathrm{~g} / \mathrm{dl}$, hematocrito $25,4 \%$, urea $274 \mathrm{mg} / \mathrm{dl}$, creatinina 11,11 $\mathrm{mg} / \mathrm{dl}$, filtrado glomerular $5 \mathrm{ml} / \mathrm{min} / 1,73 \mathrm{~m}^{2}$, calcio 7,8 $\mathrm{mg} / \mathrm{dl}$, fósforo $6,9 \mathrm{mg} / \mathrm{dl}$, potasio $4,7 \mathrm{mmol} / \mathrm{L}$, sodio 138 $\mathrm{mmol} / \mathrm{L}$, albúmina $3,2 \mathrm{~g} / \mathrm{dl}$, proteínas en orina $1357 \mathrm{mg}$ en 24 horas, IgG $701 \mathrm{mg} / \mathrm{dl}$, IgA $519 \mathrm{mg} / \mathrm{dl}$, IgM 43 $\mathrm{mg} / \mathrm{dl}$, Alfa-1 globulina 6,2\%, Alfa-2 globulina 10,9\%, Beta globulina 20,1\%, Gamma globulina $10,6 \%$ proteína C reactiva $5,30 \mathrm{mg} / \mathrm{dl}$, en el mielograma se observan hallazgos citológicos compatibles con infiltración $>10 \%$ de células plamáticas ( $16 \%$ ) y citometría de flujo del 5,54\%.

Tras esta analítica y aspirado medular se le diagnostica un mieloma múltiple IgA Lambda oligosecretor de cadenas ligeras IPSS3 y DS III y fracaso renal agudo con marcada proteinuria de Bence Jones. Se indica tratamiento de quimioterapia según esquema VTD (Velcade, Talidomida, Dexametasona), junto con terapia de hemodiálisis con filtro de alto poro que se inicia el 9 de agosto 2016.

Para realizar la técnica de hemodiálisis se colocó un catéter venoso central tunelizado (CVT) yugular que proporcionó un flujo suficiente $(250 \mathrm{ml} / \mathrm{min})$, ya que para este tipo de terapia sólo se requiere de una depuración lenta. Durante la hemodiálisis se utilizó un flujo de baño de $500 \mathrm{ml} / \mathrm{min}$.

Se le realizaron 6 sesiones de hemodiálisis diarias con el dializador de alto poro, la primera sesión fue de 6 horas y el resto de las sesiones fueron de 8 horas. Posteriormente continúo con sesiones de hemodiálisis 2-3 días a la semana, con diálisis convencional con dializador de helixone ${ }^{\circledR}$ plus, hasta el 30 de agosto 2016.

Todos los días (durante las 6 sesiones) antes de realizar la conexión al circuito extracorpóreo se le administraba 1 ampolla de polaramine $5 \mathrm{mg}$ IV y $100 \mathrm{mg}$ de hidrocortisona IV, para evitar reacciones anafilácticas al dializador y al circuito extracorpóreo.

En cada sesión de hemodiálisis se administraban 3 viales de albúmina humana $20 \%$, uno al inicio de la sesión y dos viales al final de la misma. Con el objetivo de evitar la hipocalcemia también se administran 2 ampollas de gluconato cálcico al final de la sesión. El sellado del catéter se realizaba con heparina sódica $1 \%$, según protocolo.

En la última sesión fue necesario administrar dos concentrados de hematíes, tras detectar en la analítica de sangre una hemoglobina de $8,5 \mathrm{~g} / \mathrm{dl}$.

Todo esto permitió que mejorará el filtrado glomerular a $17 \mathrm{ml} / \mathrm{ml} / 1,73 \mathrm{~m}^{2}$, posiblemente porque se iniciaron las sesiones de hemodiálisis precozmente. La evolución de las cadenas ligeras se muestra en la Figura 1.

Evolución de las cadenas ligeras por sesión de HD Lambda post (mg/dl) Lambda pre (mg/dl)

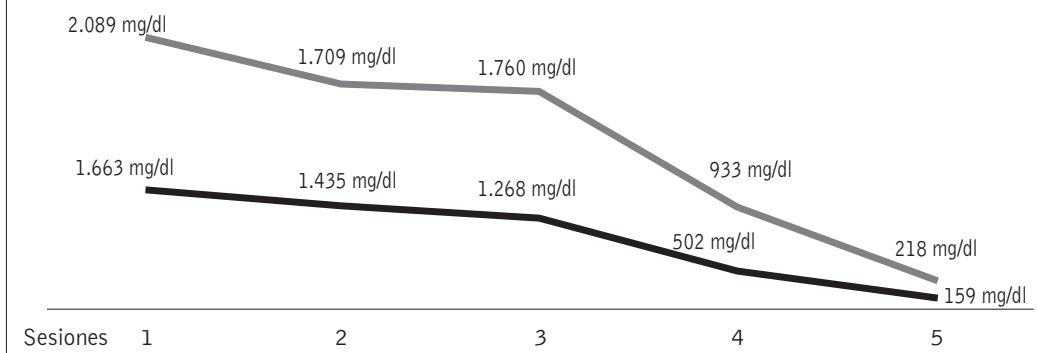

Figura 1: Caso 1. Evolución cadenas ligeras tras las sesiones.

Durante las sesiones de hemodiálisis se monitorizó la tensión arterial, que se mantuvo estable. También se controlaron la presión arterial y venosa del circuito, manteniéndose un flujo de sangre de $250 \mathrm{ml} / \mathrm{min}$. 0bservamos una caída de presión transmembrana coincidiendo con coagulaciones del circuito, siendo más frecuente durante las primeras sesiones, por lo que se ajustó la dosis de heparina a 2000UI en bolo al inicio de la sesión y $5000 U$ I en perfusión continúa.

Se extrajo analítica de sangre al inicio y al final de cada sesión (hemograma y bioquímica), para valorar la disminución de las cadenas ligeras y ajustar los aportes de albúmina, gluconato cálcico, potasio y fósforo, entre otros.

El paciente mostró una buena respuesta y aceptación a la terapia, y se dializó en una cama en vez de sillón para facilitar su confort. Dado su buen estado general, se le dio de alta hospitalaria el 12 de agosto, indicándo- 
se controles analíticos en las sesiones de hemodiálisis y seguimiento en consulta de hematología.

A los 6 meses de haber finalizado el tratamiento combinado se apreciaron los siguientes valores analíticos: hemoglobina $11,2 \mathrm{~g} / \mathrm{dl}$, hematocrito $33,7 \%$, urea 60 $\mathrm{mg} / \mathrm{dl}$, creatinina $2,45 \mathrm{mg} / \mathrm{dl}$, filtrado glomerular $29 \mathrm{ml} /$ $\mathrm{min} / 1,73 \mathrm{~m}^{2}$, calcio $9,3 \mathrm{mg} / \mathrm{dl}$, fósforo $3,6 \mathrm{mg} / \mathrm{dl}$, potasio 4,6 $\mathrm{mmol} / \mathrm{L}$, sodio $141 \mathrm{mmol} / \mathrm{L}$, albúmina 4,2 g/dl, IgG $976 \mathrm{mg} / \mathrm{dl}$, IgA $212 \mathrm{mg} / \mathrm{dl}$, IgM $200 \mathrm{mg} / \mathrm{dl}$.

El día 18 de enero del 2017 se realizó autotrasplante sin complicaciones, manteniendo función renal estable. A Julio de 2017 el paciente continúa estable y controladado en la consulta de nefrología y hematología del Hospital Rey Juan Carlos (Móstoles).

\section{Caso 2}

Paciente varón de 73 años, antecedentes personales de hipertensión, múltiples lesiones líticas en calota ósea de cráneo, en mandíbula y huesos faciales, diagnosticado en 2014 de mieloma múltiple.

En septiembre del 2016 ingresa en el Hospital de Villalba por recidiva de un mieloma múltiple e inicia tratamiento de quimioterapia con VMP (Velcade, Bortezomib,Dexametasona) y Bifosfonatos. En el segundo ciclo de tratamiento, el paciente sufre un episodio de obstrucción intestinal y en el tercero se le diagnostican una lesión orbitaria izquierda que se cree puede estar en relación con su patología. Se continúa su seguimiento en el Hospital Rey Juan Carlos (Móstoles).

En enero 2017 acude a revisión de hematología y tras la analítica de sangre se observa un empeoramiento de la función renal con una creatinina $3,65 \mathrm{mg} / \mathrm{dl}$ y un filtrado glomerular de $17 \mathrm{ml} / \mathrm{min} / 1,73 \mathrm{~m}^{2}$ y le derivan a servicio de urgencias para ingreso (anteriormente en su revisiones se mantenía estable con una creatinina de $2,40 \mathrm{mg} /$ dl y un filtrado glomerular de $28 \mathrm{ml} / \mathrm{min} / 1,73 \mathrm{~m}^{2}$ ).

En la analítica de sangre se observa: hemoglobina $10,7 /$ $\mathrm{dl}$, hematocrito $38 \%$, urea $104 \mathrm{mg} / \mathrm{dl}$, creatinina 3,38 $\mathrm{mg} / \mathrm{dl}$, filtrado glomerular $19 \mathrm{ml} / \mathrm{min} / 1,73 \mathrm{~m}^{2}$, potasio 4,1 mmol/L, sodio $130 \mathrm{mmol} / \mathrm{L}$, albúmina $2,5 \mathrm{~g} / \mathrm{dl}, \mathrm{IgG}$ $299 \mathrm{mg} / \mathrm{dl}$, IgA $33 \mathrm{mg} / \mathrm{dl}$, Ig M $29 \mathrm{mg} / \mathrm{dl}$. B2 microglobulina $10,6 \mathrm{mg} / \mathrm{l}$.

El 19 de enero 2017 se iniciaron sesiones diarias de hemodiálisis con dializador de poro ancho. Se realizaron 5 sesiones, siendo la primera de 6 horas y el resto de las sesiones de 8 horas consecutivas. Se utilizó en todas las sesiones un flujo de baño de $500 \mathrm{ml} / \mathrm{min}$.

Para realizar la técnica de hemodiálisis se colocó un catéter femoral no tunelizado, el cúal planteó problemas de flujo (200-250 ml/min), por lo que se tuvieron que interrumpir dos sesiones para administrarle un bolo de urokinasa 50.000UI por rama para desobstruir el catéter venoso central. Tras la sesión de diálisis se sellaba el catéter con urokinasa 25.000 U por rama.

Durante las sesiones de hemodiálisis se mantuvo estable la tensión y se le administró heparina sódica $1 \%$ intradiálisis en una dosis inicial de 1.500 U y 5.000 U en perfusión continúa.

Se le extrajo analítica de sangre al inicio y al final en la primera, segunda y quinta sesión para valorar la disminución de las cadenas ligeras, filtrado glomerular, y realizar los aportes necesarios de albúmina y gluconato cálcico.

Le administramos en todas las sesiones 1 vial de albúmina IV y 2 ampollas de gluconato cálcico IV post- hemodiálisis.

El paciente obtuvo buena respuesta al tratamiento $y$ aceptación a la terapia, se dializó en cama para facilitar el confort del paciente.

Todo esto permitió que mantuviera el filtrado glomerular aunque no mejoró, probablemente por tratarse de un caso de mieloma recidivante. La evolución de las cadenas ligeras se muestra en la Figura 2.

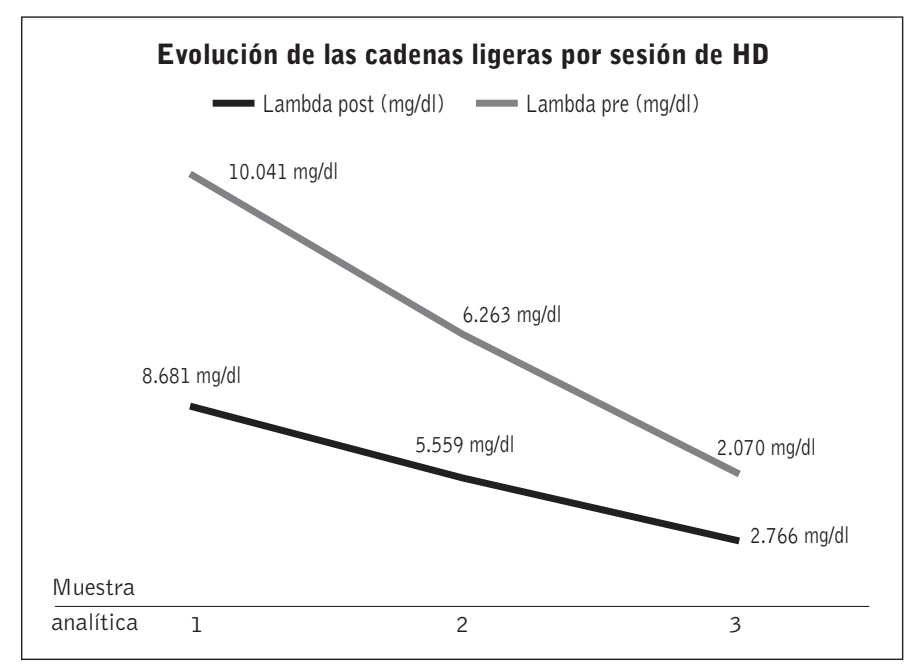

Figura 2: Caso 2 Evolución de las cadenas ligeras tras las sesiones. 
Un mes más tarde, se dio de alta hospitalaria, presentando los siguientes valores analíticos: hemoglobina $9,4 / \mathrm{dl}$, hematocrito $29,3 \%$, urea $59 \mathrm{mg} / \mathrm{dl}$, creatinina $2,75 \mathrm{mg} / \mathrm{dl}$, filtrado glomerular $24 \mathrm{ml} / \mathrm{min} / 1,73 \mathrm{~m}^{2}$, calcio $6,6 \mathrm{mg} / \mathrm{dl}$, fósforo $2,7 \mathrm{mg} / \mathrm{dl}$, potasio $3,5 \mathrm{mmol} / \mathrm{L}$, sodio $137 \mathrm{mmol} / \mathrm{L}$, albúmina 2,3/dl, IgG $327 \mathrm{mg} / \mathrm{dl}, \operatorname{IgA}$ $34 \mathrm{mg} / \mathrm{dl}$, IgM 31 mg/dl. B2 microglobulina 4,1 mg/l.

A mayo del 2017 el paciente continúa estable y controlado en la consulta de nefrología y hematología del Hospital rey Juan Carlos (Móstoles).

\section{Discusión}

Como referencia a la literatura, la hemodiálisis de larga duración con filtros para eliminar cadenas ligeras (alto cut-off) se postula como el tratamiento más eficaz para la nefropatía del mieloma. Nuestra experiencia en los dos casos presentados, tratados con hemodiálisis larga con Filtryzer BK-2.1 ${ }^{\circledR}$, se ha observado un claro descenso de las cadenas ligeras en cada sesión con buena tolerancia y recuperación de la función renal en un caso. Creemos que el realizar precozmente las sesiones de hemodiálisis fue uno de los factores determinantes para obtener un resultado positivo.

Como refieren otros autores, la coagulación del circuito es un problema frecuente ${ }^{12-13}$, en nuestros pacientes, también observamos la facilidad con que se coagulaba el circuito, agravado en uno de los pacientes por el malfuncionamiento del catéter.

Respecto a la eficacia de la hemodiálisis, con un flujo de sangre de $250 \mathrm{ml} / \mathrm{min}$ y un flujo de líquido de diálisis de $500 \mathrm{ml} / \mathrm{min}$ aconsejado por otros autores ${ }^{12-15}$, se consiguió un buen aclaramiento de cadenas ligeras y una mínima pérdida de albúmina. Respecto a la pérdida de albúmina, pudimos comprobar en nuestros pacientes que la infusión de 2 viales de albúmina humana al $20 \%$ hacia el final de la diálisis permitía mantener unos niveles aceptables de 2,58 g/dL de media después de 6 sesiones de tratamiento, frente a los 2,94 del inicio. Al finalizar el tratamiento, la albúmina había mejorado hasta niveles de $2,75 \mathrm{~g} / \mathrm{dL}$.

Otro aspecto a comentar es el de los $\operatorname{costos}^{11,12}$, que la literatura define como un inconveniente si se compara con una hemodiálisis convencional, ya que debe administrarse albumina y utilizar un filtro especial, ya que otros aspectos como personal y otro material son similares. En nuestro caso se han disminuido los costos utilizando
Filtryzer BK-2.1 ${ }^{\circledR}$, frente a otros filtros especiales existentes en el mercado.

Consideramos que es muy importante el seguimiento estrecho de esta patología y un inicio temprano del tratamiento ante cualquier alteración de la función renal. En nuestra experiencia, la combinación del tratamiento con quimioterapia y diálisis con filtro Filtryzer BK-2.1 ${ }^{\circledR}$ ha sido una buena opción para la mejora la función renal de estos pacientes afectos de mieloma múltiple.

\section{Agradecimientos}

Especialmente a Dra. Laura García- Puente, Dra. Simona Alexandru, José María Bueno Fuentes, Antonio Ochando García, Gema Sánchez de León y Francisco Jesús Horrillo, por toda la ayuda facilitada.

Recibido: 15 agosto 2017

Revisado: 4 septiembre 2017

Modificado: 7 abril 2018

Aceptado: 30 mayo 2018

\section{Bibliografía}

1. García-Sanz R, Mateos MV, San Miguel JF. Mieloma múltiple. Mes Crin (Barco) 2007;129(3):104-15.

2. Hutchinson CA, Bateman $V$, Behrens $J$ et al: The pathogenesis and diagnosis of acute kidney injury in multiple myeloma. Nata Rev. Nephrol 2012; 8:4351.

3. Hutchison C.A, Plant T, Drayson M, Cockwell P, Basnayake $\mathrm{K}$ et al.Serum free light chain measurement ainnds the diagnosis of myeloma in patients with severe renal failure.BMC Nephrol. 2008;9(1):11.

4. Basnayake K, Stringer S, Hutchison C, Cockwell P. The Biology of immunoglobulin FLC and kidney injury. Kidney Int 2011;79: 1289-01.

5. Gaballa MR, Laubach JP, Schlossman RL, Redman K, Noonan K, Mitsiades CS et al. Management of myeloma-associated renal dysfunction in the era of novel therapies. Expert Rev Hematol 2012;5:51-66. 
6. Clark WF, Stewart AK, Rock GA, Sternbach $M$, Sutton DM, Barrett BJ et al. Plasma exchange when myeloma presents as acute renal failure: a randomized, controlled trial. Ann Intern Med 2005;143:777-84.

7. Álvarez-Lara M.A, Martín-Malo A, Aljama-García P. Nuevas alternativas en el tratamiento del riñón del mieloma Nefrologia. 2013;33:443-7.

8. Levy F, Camarero termiño V, Torres Torres G, Hijazi Prieto B. Plasmaféresis como tratamiento adyuvante en el fallo renal agudo secundario riñón del mieloma. Nefrología. 2009;29(3):283-284.

9. Lahoz VC, Zulaica EPR, Soler AV, Letosa M M, Buil BA. El fracaso renal agudo por mieloma múltiple: Cuidados de los pacientes en una unidad de hemodiálisis. Rev la Soc Esp Enferm Nefrol.17(2):135-43.

10. Martín-Gómez M.A, García-Marcos, Caba-Molina S.A.M, Palacios-Gómez M.E, Gómez-Morales M, Claver-Ferré C. Riñón de mieloma: importancia de la valoración de respuesta mediante monitorización de cadenas ligeras libres en suero. Nefrologia 2013;33:862-4.

11. Borrego Hinojosa J, Pérez-del Barrio M.P, Biechy-Baldan M, Merino-García E, Sánchez Perales M.C, García Cortés M.J et al. Tratamiento con hemodiálisis larga con filtros de alto cut-of en la nefropatía por cilindros del mieloma: nuestra experiencia. Nefrologia 2013;33:515-23.
12. Berni Wennekers A, Martín Azara M.P, Dourdil Sahu V, Bergasa B, Ruiz Laiglesia JE, Vernet $P$ et al. Trece tratamientos de la insuficiencia renal aguda secundaria a mieloma múltiple con filtros de high cut off. Nefrologia. 2016;36:418-26.

13. Testa $A$, Dejoie $T$, Lecarrer $D$, Wratten $M$, Sereni $L$, Renaux JL. Reduction of free inmunoglobulin light chains usingadsorption properties of hemodiafiltration with endogenous reinfusion. Blood Purif. 2010;30:34-6.

14. Martin Reyes G, Tolerdo-Rojas R, Torres-Rueda A, Sola -Moyano E, Blanca- Martos L, Fuentes-Sanchez $L$ et al. Tratamiento del fracaso renal agudo en el riñón del mieloma con hemodiálisis utilizando filtros de poro grande (Hight Cut -off). Nefrologia. 2012;32(1):35-43.

15. Pendón-Ruiz de Mier M.V, Álvarez-Lara M.A, 0jeda-López R, Martin-Malo A, Carracedo J, Caballero - Villarraso $\mathrm{J}$ et al. Eficacia de la hemodiálisis con regeneración del ultrafiltrado en la reducción de cadenas ligeras en mieloma múltiple con insuficiencia renal. Revista Nefrología.2013; 33(6):788-96.

Este artículo se distribuye bajo una Licencia Creative Commons Atribución-NoComercial 4.0 Internacional. https://creativecommons.org/licenses/by-nc/4.0/

Open Access (c) $($ ) $(9)$ 